tents into a sarcophagus of solid granite. Beyond it lies the zone of metamorphosed rock, and beyond that, a final zone where hydration reactions will add a final barrier by plugging rock fractures. These zones constitute a multiple "near field" barrier, whereas the 4-km burial depth-much greater than that envisaged for conventional nuclear repository-constitutes the "far field" barrier.

Gibbs said that rocks are much more uniform at such great depth, liberating planners from the dictates of shallow geology, and thus greatly increases the choice of available sites. He said also that any intra-rock water present at these depths is stable, has been there for millions of years, and will not return to surface so long as the hole is adequately back-filled and sealed. Leakage of radionuclides from the sarcophagus would probably not matter since the waters present at such depths have not moved for millions of years. Very deep disposal of this sort also increases the likelihood of the containment surviving earthquakes.

\section{Hybrid Process Uses Electromagnetic Force to Mold Aluminum Auto Parts}

Glenn S. Daehn, professor of materials science and engineering at Ohio State University, and his colleagues at Ohio State and General Motors developed a process that combines traditional metal stamping with electromagnetic forming. In this hybrid technique, a tool stamps out the general shape of a part, and electromagnetic pulses help form details.

As Vincent J. Vohnout, a postdoctoral researcher in materials science and engineering, reported at the International Conference on Technology of Plasticity in Nuremberg, to keep the aluminum sheet from tearing, the researchers softened the shape of the tool, replacing sharp corners with gradual curves, and stamped the general shape of the part. They then turned to electromagnetic forming, and like-charged metal coils repel the metal into a mold. The researchers placed coils behind only the unfinished spots of the panel, and used electromagnetic force to

\section{Biomechanics Meeting Presents Materials-Related Topics}

Scientists at the 23rd Annual Meeting of the American Society of Biomechanics, held in October 1999 in Pittsburgh, presented materials-related issues involving polymers, surface treatment, and bone prostheses. In one presentation, by simulating the natural load on human thigh bones, scientists Michael H. Santare and Suresh G. Advani of the University of Delaware proposed a design for an artificial hip that would reduce stress-shielding and prevent bone atrophy. After they modeled the function of a healthy hip, Santare and Advani compared their findings with an analysis of the stress patterns produced by a conventional artificial hip and by the design pioneered by $\mathrm{E}$. Munting and M. Verhelpen in 1995 in which a much shorter rod than previously used, attached to the top of the thigh by several small bolts, was inserted through the exterior side of the bone.

Santare and Advani's resulting design greatly reduced the prosthetic rod, which seems to be responsible for much of the rigidity and altered loading that occurs following hip replacement. In its place, they used a system of cables to fix the implant to the proximal femur. A platelike base is used to transfer the bending loads from the prosthesis to the bone. Santare said that further improvement can be achieved by using advanced composite materials to tailor the stiffness of the implant. While the design was still in prototype form during the conference, Santare said that mechanical tests using bone from a cadaver suggested the viability of this new technology.

To address the threat of aseptic loosen- ing to the long-term success of cemented total hip stems, Keith T. Hustosky's research team at West Virginia University studied the pull-out loads and axial displacements of stems that differed only in surface roughness to determine the effects of surface finish on total hip-stem behavior. Using PMMA bone cement to insert tapered, collarless total hip stems into nine femora, the researchers tested seven specimens with a polished-surface finish and two with a matte-surface finish. They applied a tensile axial force to the stems through a pull-out fixture. With the mean pull-out load of $1238 \mathrm{~N}$, the polished stems were extracted from the cement mantle without damaging the cement, bone, or fixture. The researchers were unable to pull the stems with a matte finish out of the cement without the femur fracturing. They reported a mean failure load of $6743 \mathrm{~N}$.

When analyzing three stem-cement interface conditions-perfect bond, debonded with a friction coefficient of 0.22 , and completely debonded-the researchers found that "the matte-finish specimens achieved a well-bonded stemcement interface, while the polished specimens had debonded at the stemcement interface with a friction coefficient less than 0.22." They reported that failure of the bone occurred before failure of the interfaces and cautioned that study of the pull-out strengths should not be the only means of evaluating total hip stems with different surface finishes.

Researchers at the Biomechanics Laboratory in the Minneapolis Sports Medicine Center and Cardiac Assist Technologies in Pittsburgh have deter- mined that low-density polyurethane (LDPU) foam models can be used to characterize suture pull-through properties in bone. The researchers evaluated LDPU foam with a density of $0.24 \mathrm{~g} / \mathrm{cm}^{3}$ and high-density (HDPU) foam with a density of $0.48 \mathrm{~g} / \mathrm{cm}^{3}$, representing low, to mid-range density values for cancellous bone. They recorded failure mode and load-elongation data and compared these measurements with those gathered for a comparable study using ribs. The researchers concluded that given that suture pull-through is principally a concern in patients with lower-density cancellous bone, LDPU specimens should be used to assess the pullthrough properties.

Researchers in Seoul from the Korea Institute of Science and Technology, Hansung University, Kyoung Hee University, and Hallym University determined that methodically coating a dental implant with a viscoelastic polymer can reproduce the periodontal ligament's function to absorb the shock of impact, reducing stress on the bone. The researchers coated implants with 50and 100- $\mu \mathrm{m}$ layers of chitosan-similar to a periodontal ligament's thicknessthen mechanically tested and compared initial stability between samples of coated and uncoated implants. They reported that "uncoated implants had an average shear stiffness of $34.728 \mathrm{~kg} / \mathrm{mm}$ and the coated implants $47.108 \mathrm{~kg} / \mathrm{mm}$." They reported that the shear stiffness of a coated implant is increased by $35.64 \%$ because the material expanded, filling the gap between the implant and the alveolar bone during the implant operation. 
push the panel all the way into the sharp corners of the original mold.

In tests, the researchers were able to form aluminum door panels that closely matched the shape of their steel counterparts without tears or wrinkles. They also performed formability tests on both agehardened and non-hardenable aluminum-two types of the metal used commercially-and found both exhibited tremendous gains with this process.

While traditional stamping can stretch most aluminum alloys to a maximum of $30 \%$ of their original length before tearing, Daehn and colleagues have stretched parts a full $100 \%$ without tearing. Also, tests have shown this process does not degrade the material.

"That extra elongation enables us to form many complicated parts that are otherwise difficult to impossible," Daehn said.

These tests were performed with two steps, first the stamping and then the electromagnetic forming. But Daehn envisions that manufacturers could install electromagnet coils within a die and perform both steps at the same time. According to this scenario, when the mold closes, the aluminum will first conform to the softer shape of the tool. Then, the electromagnetic force from the coils will push the metal the rest of the way into the die. As Daehn describes it, the part would "pop into shape."

Daehn said manufacturers already use electromagnetics for assembly, but not for sheet-metal forming.

\section{Hydrogen Peroxide could Power Future Fuel Cell}

Researchers at Purdue University are developing a hydrogen peroxide-based material into a type of environmentally friendly fuel cell that runs on aluminum and renewable resources and generates about 20 times more electricity per pound than car batteries. Stephen Heister, a professor of aeronautics and astronautics at Purdue, said that the mixture consists of $3 \%$ water and $97 \%$ hydrogen peroxide and has had its critical contaminants removed.

As described in a poster presented at the Second International Hydrogen Peroxide Propulsion Conference at Purdue, this purified, concentrated form of $\mathrm{H}_{2} \mathrm{O}_{2}$ is broken down with chemical catalysts, yielding oxygen that combusts with alcoholbased fuels such as methanol or ethanol, which can be derived from corn. The researchers said that in the fuel cells, hydrogen peroxide serves two roles: It is a "catholyte," meaning it is both the electrolyte and the cathode. The aluminum serves as the cell's fuel and its anode; as it oxidizes, it gives up electrons. Waste products include water and recyclable chemical compounds.

In the past, attempts to develop the cells were abandoned because the reaction with aluminum quickly formed a thick sludge that hindered the flow of electricity. However, because Heister and his colleagues did not have pure aluminum for their work, they used an aluminum alloy and found that the alloy did not form the sludge.

Engineering student Kok Hong Lim said that one problem with the experimental cells is that, unlike batteries, they do not immediately provide a steady supply of electricity; the cells take about $2 \mathrm{~h}$ to reach their peak electrical output before producing a steady current flow.

John Rusek, an assistant professor of aeronautics and astronautics at Purdue, said that aluminum was chosen originally because it is an abundant natural resource and is readily available from recycled sources. He estimates that the cells are at least 20 times higher in energy density than a standard lead-acid car battery. He said that other metals, such as lithium alloys, might also work in hydrogenperoxide fuel cells.

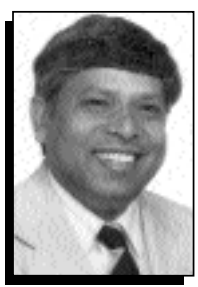

\section{ASM Gold Medal} Awarded to Narayan

Jagdish Narayan, Distinguished University Professor of Materials Science and Engineering at North Carolina State University, is the recipient of the 1999 ASM Gold Medal, the highest honor given by ASM-International, for his seminal contributions in the area of laser processing, defects and interfaces, structure-property correlations, and modeling of novel and advanced materials. He was honored on November 2 during the ASM-International's annual meeting in Cincinnati.

Narayan joined the University in 1984, where he directs the NSF Center for Advanced Materials and Smart Structures, after working as a senior scientist and group leader for 12 years at Oak Ridge National Laboratory. He received his master's (1970) and PhD degree (1971) from the University of California-Berkeley, and his bachelor's degree with highest honors and distinction from the Indian Institute of Technology, India, in materials science and engineering. He has published over 700 papers, edited eight books, and holds 15 patents. Narayan served on the Materials Research Society council (1984-1987) and co-chaired the 1984 MRS Fall Meeting. He is a member of MRS and a Fellow of the American Physical Society; the American Association for the Advancement of Science; the Minerals, Metals \& Materials Society; and ASM-International.

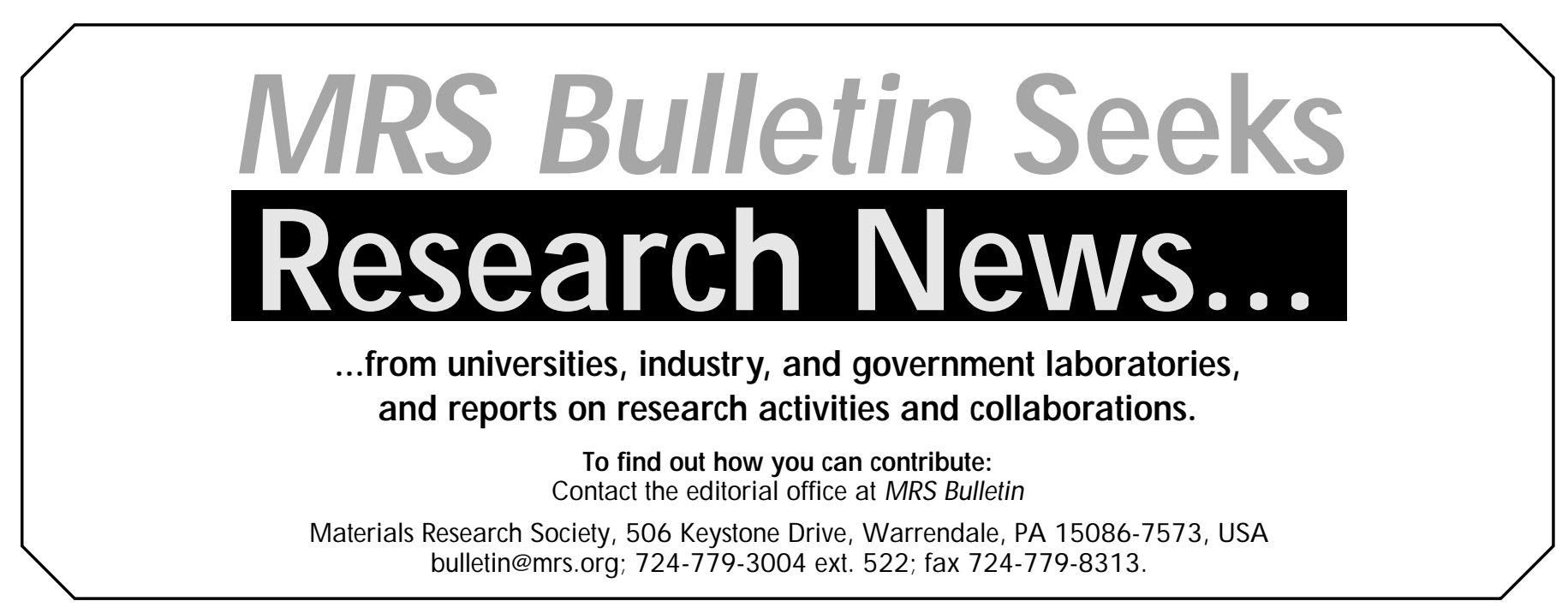

